\title{
Fibroblast Growth Factor 23 and $\alpha$-Klotho Protein Are Associated with Adverse Clinical Outcomes in Non-Dialysis CKD Patients
}

\author{
Eleni Manou ${ }^{a}$ Elias Thodis $^{b}$ Georgios Arsos $^{c}$ Ploumis Pasadakis ${ }^{b}$ \\ Stylianos Panagoutsos ${ }^{b}$ Dorothea Papadopoulou ${ }^{a} \quad$ Aikaterini Papagianni $^{d}$ \\ aDepartment of Nephrology, Papageorgiou Hospital, Thessaloniki, Greece; 'bepartment of \\ Nephrology, Democritus University of Thrace, Alexandroupolis, Greece; '3rd Department of \\ Nuclear Medicine, Aristotle University of Thessaloniki, Papageorgiou Hospital, Thessaloniki, \\ Greece; dDepartment of Nephrology, Aristotle University of Thessaloniki, Hippokration \\ Hospital, Thessaloniki, Greece
}

\author{
Keywords \\ Chronic kidney disease · Fibroblast growth factor $23 \cdot$ Soluble $\alpha$-Klotho $\cdot$ Mortality
}

\begin{abstract}
Background: Fibroblast growth factor 23 (FGF-23) and $\alpha$-Klotho protein appear to have an important role in the pathogenesis of CKD-mineral and bone disorders. The aim of this study was to investigate the association of FGF-23 and $\alpha$-Klotho levels with adverse clinical outcomes in patients with non-dialysis CKD. Materials and Methods: We conducted a prospective cohort study, enrolling participants with non-dialysis CKD from a single center in Greece. At enrollment, glomerular filtration rate (GFR) was measured (mGFR) and plasma levels of carboxyl terminal FGF-23 (cFGF-23) and soluble $\alpha$-Klotho (sKlotho) were determined by enzyme-linked immunoassay. Participants were followed for up to 5 years or until the occurrence of the primary endpoint of initiation of renal replacement therapy or death. Multivariate regression tree analysis was used to identify informative baseline parameters in order to categorize participants. Also, using median values of CFGF-23 and sKlotho, participants were categorized into 4 groups, in whom survival was compared using Kaplan-Meier and Cox regression analysis. Results: 128 participants were enrolled with a median mGFR of $41.5 \mathrm{~mL} / \mathrm{min} / 1.73 \mathrm{~m}^{2}$ $(\mathrm{IQR}=28.2)$. Baseline mGFR correlated with cFGF-23 and sKlotho $(r=-0.54$ and $r=0.49$, respectively; $p<0.0001$ for both). cFGF-23 and sKlotho levels correlated negatively $(r=-0.24$, $p=0.006)$. Multivariate regression tree analysis resulted in 3 groups defined by cutoff values of $\mathrm{mGFR}\left(60.9 \mathrm{~mL} / \mathrm{min} / 1.73 \mathrm{~m}^{2}\right)$ and phosphate $(3.7 \mathrm{mg} / \mathrm{dL})$. These groups correlated with CKD stage, cFGF-23, and sKlotho ( $p<0.0001$ for all). During a median follow-up of 36 months
\end{abstract}


$(I Q R=22), 40$ (31.2\%) participants reached the primary endpoint (31 initiated renal replacement therapy, 9 died). Survival to primary endpoint differed among the 4 groups formed using median values of both biomarkers, with the low FGF-23/high Klotho and high FGF-23/low Klotho having the longest and shortest survival, respectively. High FGF-23/low Klotho group, compared to the opposite one, had a significantly elevated risk of the primary outcome (HR, 6.8; $95 \% \mathrm{Cl}, 2.3-19.6 ; p=0.0004)$. Conclusions: In patients with CKD stages $1-5$, the combination of higher CFGF-23 and lower sKlotho levels along with mGFR and serum phosphate was associated with adverse clinical outcomes. The utility of combinations of traditional and novel biomarkers to predict outcomes warrants further study.

\section{Introduction}

Chronic kidney disease-mineral and bone disorder (CKD-MBD) is a spectrum of pathologies accompanying CKD and worsening in parallel with the deterioration in renal function. Recent evidence suggests that alterations of fibroblast growth factor 23 (FGF-23) and $\alpha$-Klotho protein are the earliest detectable biochemical abnormalities of CKD-MBD [1-3].

FGF-23 is produced by osteoblasts and osteocytes and induces a negative phosphate balance by functioning as a phosphaturic peptide as well as a counterregulatory hormone for calcitriol, leading to reduced intestinal absorption of phosphate. The relationship between secondary hyperparathyroidism (SHPT) and FGF-23 is complex. FGF-23-induced inhibition of calcitriol contributes to SHPT development, while FGF-23 also inhibits parathyroid hormone (PTH) production and secretion, thus opposing the progression of SHPT [4-6]. Beginning in early CKD, FGF-23 progressively increases, reaching up to 1,000-fold higher than normal in patients with end-stage kidney disease [5]. Accumulating evidence in CKD patients also suggests that excess FGF-23 exerts important actions on cardiovascular system and contributes to the increased cardiovascular morbidity and mortality observed in this population [7-14].

$\alpha$-Klotho is mainly produced in the kidney and functions as an obligatory co-receptor for FGF-23, in its classic target organs $[15,16]$. There are 2 basic forms of $\alpha$-Klotho protein, a membrane-bound peptide and a soluble protein generated from extracellular domain cleavage by 2 membrane-anchored proteases (secretases) [16]. Via glucosidase or sialidase activity, soluble $\alpha$-Klotho (sKlotho) acts to suppress growth factor signaling, protects from oxidative stress, and regulates a variety of ion channels and transporters [15, 17]. $\alpha$-Klotho also has a key role in phosphate metabolism as a phosphaturic factor, independent of its role as a co-receptor of FGF-23 [18]. During the last decade, $\alpha$-Klotho has gained the attention of the nephrology community, as an early biomarker of either acute or chronic kidney injury [17]. In contrast to FGF-23, $\alpha$-Klotho plasma levels in CKD show a progressive decrease in parallel with CKD progression [16]. However, it is not clear at which level of glomerular filtration rate (GFR), this decrease becomes obvious. Moreover, the number of studies investigating the probable association of $\alpha$-Klotho levels with adverse clinical outcomes is limited and provides contradictory results, with one study suggesting that low $\alpha$-Klotho levels are related to deterioration of renal function and increased mortality [19] and other studies failing to confirm these findings [20]. The aim of the present study was to investigate the associations of FGF-23 and $\alpha$-Klotho levels with adverse clinical outcomes and mortality in patients with non-dialysis CKD stages 1-5. 


\section{Materials and Methods}

\section{Patients}

Between February 2011 and November 2013, outpatients of the Department of Nephrology at Papageorgiou General Hospital were screened for eligibility. Inclusion criteria were age $>18$ years and CKD, defined according to criteria of the National Kidney FoundationKidney Disease Outcomes Quality Initiative 2002 for CKD staging (NKF-K/DOQI) [21]. Exclusion criteria were active inflammatory, hepatic, or malignant disease and use of corticosteroids or other immunosuppressive medication at the time of the study entry. Healthy subjects without CKD, recruited among apparently healthy blood donors of the Blood Transfusion Center at Hippokration General Hospital, served as control group to obtain reference values for carboxyl terminal FGF-23 (cFGF-23) and sKlotho.

\section{Data Collection}

Patient demographic and clinical characteristics were collected at baseline. Hypertension (HTN) was defined as a systolic blood pressure of $140 \mathrm{~mm} \mathrm{Hg}$ or more or/and diastolic blood pressure of $90 \mathrm{~mm} \mathrm{Hg}$ or more, or the use of antihypertensive drugs. Cardiovascular disease (CVD) was defined as the history of coronary artery disease or angina pectoris, ischemic/hemorrhagic stroke or transient cerebrovascular attack, and peripheral arterial disease. BMI, body surface area using the Haycock formula [22], and waist-to-hip ratio were calculated in all patients. Systolic blood pressure and diastolic blood pressure were recorded at the first visit of the patients.

Blood and urine samples were taken at baseline in the morning, under fasting conditions. A 24-h urine collection was performed in all participants, the day before the scheduled sampling. Blood samples for the measurement of cFGF-23, sKlotho, 25-hydroxy and 1,25-dihydroxy $\left(25-\mathrm{OH}\right.$ and $\left.1,25-\mathrm{OH}_{2}\right)$ vitamin $\mathrm{D}$ were separated by immediate centrifugation $(1,500 \mathrm{~g}$ for 10 $\mathrm{min}$ ), aliquoted, and stored at $-70^{\circ} \mathrm{C}$ until assay. Hematological analysis was performed in an automated hematological analyzer (ADVIA 2120i; Siemens) and biochemical tests were performed in an automated biochemical analyzer (Architect-c 16000; Abbott Diagnostics). Urine albumin, creatinine $(\mathrm{Cr}$,) and phosphate were determined from an untimed spot urine specimen. Albuminuria was determined from albumin-to-Cr ratio (ACR) in an untimed spot urine specimen and was expressed in $\mathrm{mg} / \mathrm{g}$. Also, calcium and phosphate excretion was measured in a 24-h urine collection. The ratio of tubular maximum reabsorption rate of phosphate to GFR (TmP/GFR) was also calculated in mmol/L, using serum phosphate and fractional excretion of phosphate (FEP), according to the nomogram of Walton and Bijvoet [23]. Serum intact PTH (iPTH) was assayed by the method of enhanced chemiluminescence (Immulite 2000; Siemens). Serum C-reactive protein (CRP) level was measured by nephelometry (Beckmann Coulter). Serum beta-2 microglobulin was determined by a microparticle immunoenzymatic assay (Axysm; Abbott Diagnostics). Serum levels of 25-OH and 1,25- $\mathrm{OH}_{2}$-vitamin D were determined with a radioimmunoassay method in a $\gamma$-camera counter. Plasma levels of cFGF-23 and sKlotho were measured by an ELISA, using commercially available standard kits (human cFGF-23; Immutopics Inc., San Clemente, CA, USA, and human sKlotho; Immuno-Biological Laboratories Co. Ltd, Fujioka-Shi, Japan). Plasma samples from patients were diluted 1:10 and $1: 2$, respectively, for the quantitation of cFGF-23 and sKlotho. Plasma samples from controls were tested undiluted for the quantitation of cFGF-23. The concentrations of these proteins were calculated by reference to standard curves, performed with the corresponding recombinant molecule. All plasma samples were tested in duplicate. The sensitivity of the ELISA system was $1.5 \mathrm{RU} / \mathrm{mL}$ and $6.15 \mathrm{pg} / \mathrm{mL}$ for cFGF-23 and sKlotho, respectively.

Renal function was determined by measuring GFR (mGFR) at baseline visit, using a chromium-51 EDTA ( ${ }^{51} \mathrm{Cr}$-EDTA) single-injection 2 sample radioisotopic method [24]. All 
measurements were performed between 09:00 and 13:00. Participant preparation included abstinence from xanthine-containing beverages and heavy protein intake before the test. Moderate hydration and avoidance of strenuous exercise were instructed during the procedure. Regular medications were recorded but not interrupted. A dose of $80 \mu \mathrm{Ci}{ }^{51} \mathrm{Cr}$ EDTA was given intravenously and venous blood samples were obtained from the contralateral arm at 2 and $4 \mathrm{~h}$ post-injection. The activity of $1 \mathrm{~mL}$ of plasma from each sample and of $1 \mathrm{~mL}$ of a standard solution was measured in a well-type $\gamma$-counter. GFR, in $\mathrm{mL} / \mathrm{min}$, was calculated according to the 1-compartment "slope-intercept" method with BrochnerMortensen correction for the neglected early, fast, non-renal, component of tracer plasma disappearance [25]. mGFR values were normalized for $1.73 \mathrm{~m}^{2}$ of body surface area and expressed as $\mathrm{mL} / \mathrm{min} / 1.73 \mathrm{~m}^{2}$.

\section{Follow-Up and Primary Endpoint}

The primary endpoint of the study or event was a composite of initiation of renal replacement therapy or death. After initial assessment, patients were followed up for up to 5 years. Each death was reviewed, all available medical information was collected including hospitalization records, and an underlying cause was assigned. In the case of an out-ofhospital death, family members were interviewed by telephone to better ascertain the circumstances surrounding death. Sudden death was defined as unexpected natural death within $1 \mathrm{~h}$ from symptom onset and without a prior condition that would appear fatal. At the end of follow-up, an estimated value of GFR was also recorded, characterized as final GFR (fGFR), using the CKD-EPI equation [26]. For participants who experienced the primary outcome, fGFR was derived from their serum $\mathrm{Cr}$ measurement closest to the outcome date.

\section{Statistical Analysis}

Data were reported as mean value $\pm S D$ (normally distributed data), median with range (non-normally distributed data), or frequency (percentage), as appropriate. Normality was examined by the Shapiro-Wilk test. Non-normally distributed variables were log-transformed for analyses. Correlation was assessed by the Pearson correlation coefficient, and linear regression was used to define linear relationships between 2 correlated variables. Differences in groups were assessed by Student's $t$ test or Wilcoxon-Mann-Whitney test, as appropriate. Among 3 or more groups, differences were assessed by ANOVA test or Kruskal-Wallis test, as appropriate. Contingency tables were used in order to summarize the relationships between categorical variables. Pearson's $\chi^{2}$ test was used to examine the significance of the association in contingency tables. Multiple regression analysis was used to model predictors of fGFR, using the traditional ones, which were sex, age, BMI, presence of HTN or diabetes mellitus (DM), history of CVD, waist-to-hip ratio, and hemoglobin, as well as those with significant univariate correlation with fGFR $(p<0.05)$, which were mGFR, calcium, phosphate, total alkaline phosphatase, high-density lipoprotein, ACR, CRP, FEP, TmP/GFR, iPTH, 25-OH and 1,25- $\mathrm{OH}_{2}$-vitamin D, cFGF-23, and sKlotho.

Multivariate regression tree analysis was used to determine the most "important" variables in a particular dataset [27]. In the present study, those variables were chosen from the set of variables which correlated significantly in univariate analysis with sKlotho $(p<0.05)$, namely, mGFR, hemoglobin, phosphate, ACR, FEP, calcium excretion in 24-h urine collection, iPTH, 1,25- $\mathrm{OH}_{2}$-vitamin D, and cFGF-23. This resulted in the formation of 3 distinct groups based on mGFR and serum phosphate levels. The 3 groups, named regression groups A, B, and $\mathrm{C}$, were obtained in 2 steps: the first division was made between those with an mGFR $\geq 60.85$ and with $\mathrm{mGFR}<60.85 \mathrm{~mL} / \mathrm{min} / 1.73 \mathrm{~m}^{2}$. Subsequently, the latter group was further divided into 2 other subgroups, by serum phosphate at the level of $3.7 \mathrm{mg} / \mathrm{dL}$. Thus, regression groups were as follows: A, mGFR $\geq 60.85 \mathrm{~mL} / \mathrm{min} / 1.73 \mathrm{~m}^{2}$; B, mGFR $60.85 \mathrm{~mL} / \mathrm{min} / 1.73 \mathrm{~m}^{2}$ 
and phosphate $<3.7 \mathrm{mg} / \mathrm{dL}$; and C, $\mathrm{mGFR}<60.85 \mathrm{~mL} / \mathrm{min} / 1.73 \mathrm{~m}^{2}$ and phosphate $\geq 3.7 \mathrm{mg} /$ dL.

To explore the relationship between cFGF-23, sKlotho, and patient outcomes, 4 groups were formed based on dividing the cohort by median values of cFGF-23 and sKlotho. This resulted in 4 mutually exclusive groups defined by the 4 possible combinations of lower or higher (than median) values of cFGF-23 and sKlotho.

Event-free survival was analyzed by the Kaplan-Meier analysis with relative event probability determined by the Cox proportional hazard modeling in an unadjusted and an adjusted model. The variables used as potential confounders were sex, age, BMI, waist-to-hip ratio, presence of DM, history of CVD, hemoglobin, albumin, CRP, ACR, and TmP/GFR. Additionally, mixed linear model for repeated measures was used to model GFR slope among the 4 groups. Baseline mGFR and subsequent estimated GFR values during follow-up, gathered from clinical files of our study, were plotted against time. Models were adjusted for all the above confounders. $p$ values $<0.05$ were considered statistically significant in all comparisons. Statistical analysis was performed with R, version 3.4.4 (R Foundation for Statistical Computing, Vienna, Austria).

\section{Results}

A total of 128 adult patients (86 male, median age 67 years, range 18-86) were included in this study. Baseline characteristics are reported in Table 1. At the time of study entry, 119 (93\%) were receiving 1 or more antihypertensive agents (diuretics, calcium channel blockers, angiotensin-converting enzyme inhibitors, angiotensin II receptor blockers, $\beta$-blockers, alpha-adrenergic blocking agents, and centrally acting antihypertensive agents) either for the treatment of preexisting HTN or as an anti-proteinuric or heart failure therapy, 71 (55.4\%) were receiving HMG-CoA reductase inhibitors, 57 (44.5\%) were receiving an antiplatelet agent, $28(21.9 \%)$ were receiving short- or/and long-acting insulin, and 26 (20.3\%) were receiving 1 or more oral hypoglycemic agents. None of the patients were receiving recombinant human erythropoietin therapy, phosphate binders, or other treatment for SHPT.

The relationship between baseline parameters and CKD stages is presented in suppl. Tables 1-3 (see online suppl. Tables 1-3; see www.karger.com/doi/10.1159/000510351 for all online suppl. material). A control group of 37 healthy blood donors was recruited. Mean values of cFGF-23 and sKlotho in the control group were 47.4 $\pm 53.3 \mathrm{RU} / \mathrm{mL}$ and $855.6 \pm 230.1$ $\mathrm{pg} / \mathrm{mL}$, respectively, which differed significantly from those in the study cohort $(p<0.0001$ for both). Differences were significant from stage 3 to 5 (data are not shown).

Log cFGF-23 correlated negatively with $\log$ mGFR $(r=-0.54, p<0.0001)$, log sKlotho $(r=-0.24, p=0.006)$, and $\log \mathrm{TmP} / \mathrm{GFR}(r=-0.25, p=0.004)$, and positively with log phosphate $(r=0.21, p=0.01)$ and $\log$ FEP $(r=0.46, p<0.0001)$. Log sKlotho correlated positively with $\log$ mGFR $(r=0.49, p<0.0001)$ and negatively with $\log$ phosphate $(r=-0.39, p<0.0001)$ and $\log$ FEP $(r=-0.32, p=0.0001)$. Log age correlated positively with log cFGF-23 $(r=0.22$, $p=0.009$ ) but not with log sKlotho $(p=0.104)$. Log cFGF-23 correlated positively with log waist-to-hip ratio ( $r=0.21, p=0.01)$ but not with log BMI $(p=0.503)$. cFGF-23 did not differ between patients with and without DM $(p=0.129)$ but was higher in those with a history of CVD than in those without a history of CVD $(p=0.0001)$. sKlotho was lower in participants with DM than in those without DM $(p=0.02)$ but did not differ between those with or without CVD $(p=0.416)$. Log $25-\mathrm{OH}$ and $1,25-\mathrm{OH}_{2}$-vitamin D correlated negatively with log cFGF-23 $(r=-0.26, p=0.003$, and $r=-0.34, p<0.0001$, respectively). Log sKlotho correlated positively with $\log 1,25-\mathrm{OH}_{2}(r=0.29, p=0.0007)$ but not with $25-\mathrm{OH}$-vitamin $\mathrm{D}(p=0.501)$. sKlotho did not differ between angiotensin II receptor blocker users and non-users ( $p=0.477)$, while 
Kidney and

Blood Pressure

Research
Kidney Blood Press Res 2020;45:900-915

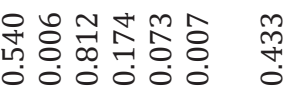

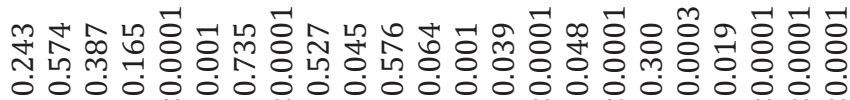
0000000

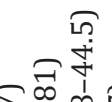

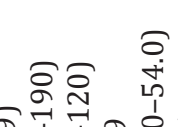<smiles>[CH-]</smiles>

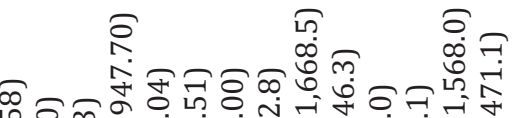
要

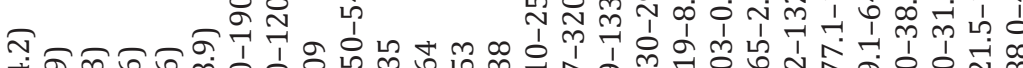

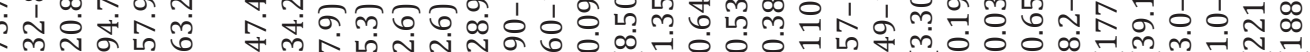

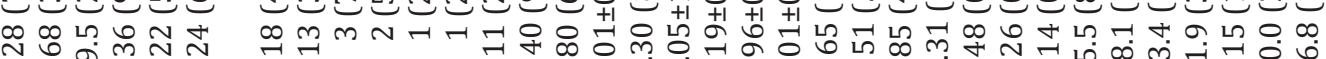

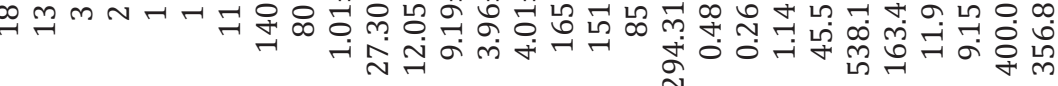

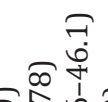

2동

$\stackrel{9}{\stackrel{2}{5}}$

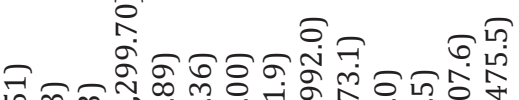

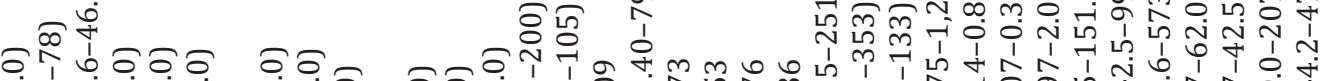

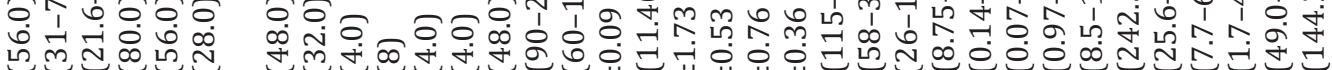

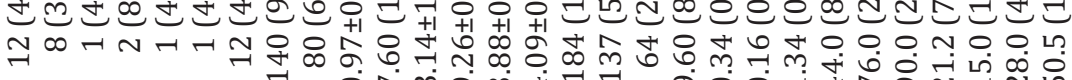
$\rightarrow \underset{\substack{\infty}}{\sim} \rightarrow$

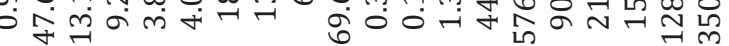

สุ

อㄴำ

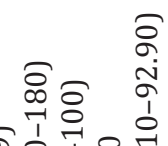

官

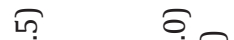

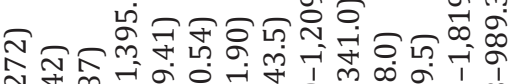
สุด

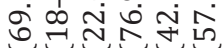

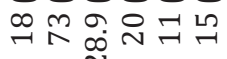

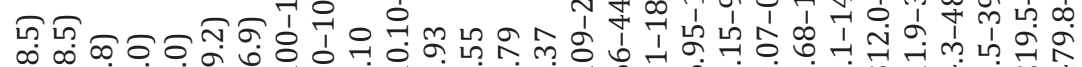

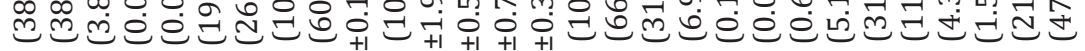
윽윽 尚

m.

ind

त席占

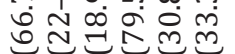

뉴욤

ळि

\&

\&

จ

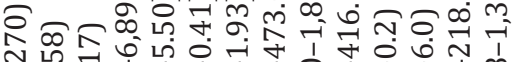
สฺศ 岂

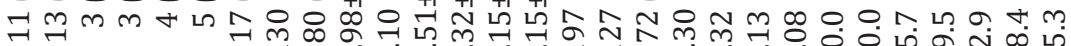
o

尺े \& î के

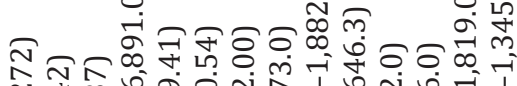
(ㄴำ 7๐

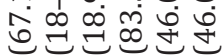
œ ๙m

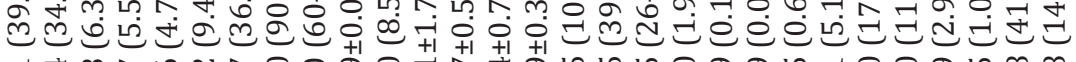

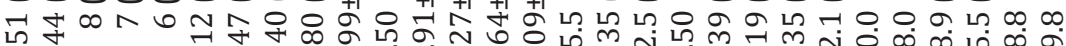

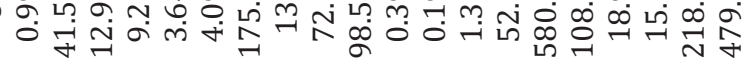

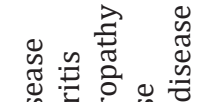

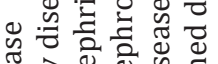

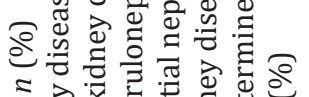

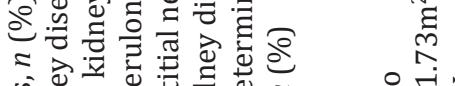

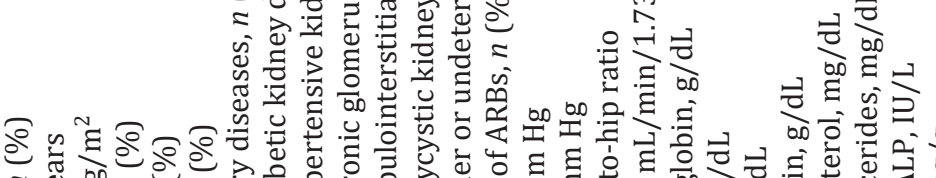

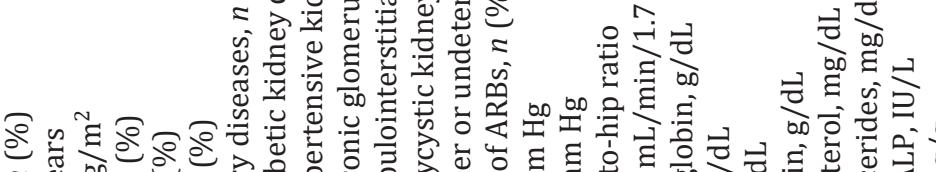

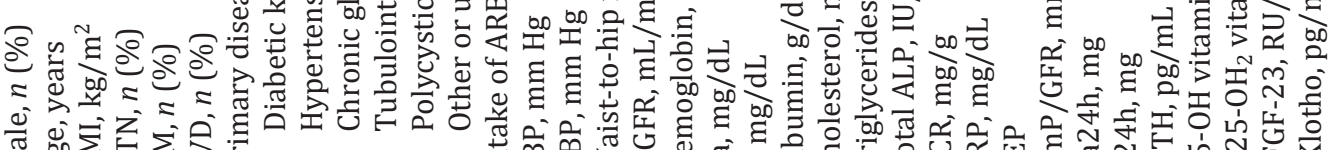

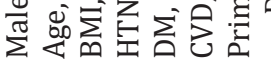

능 궁

एँ

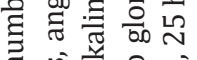

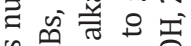

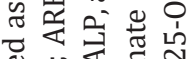

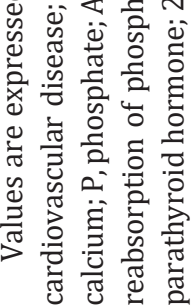




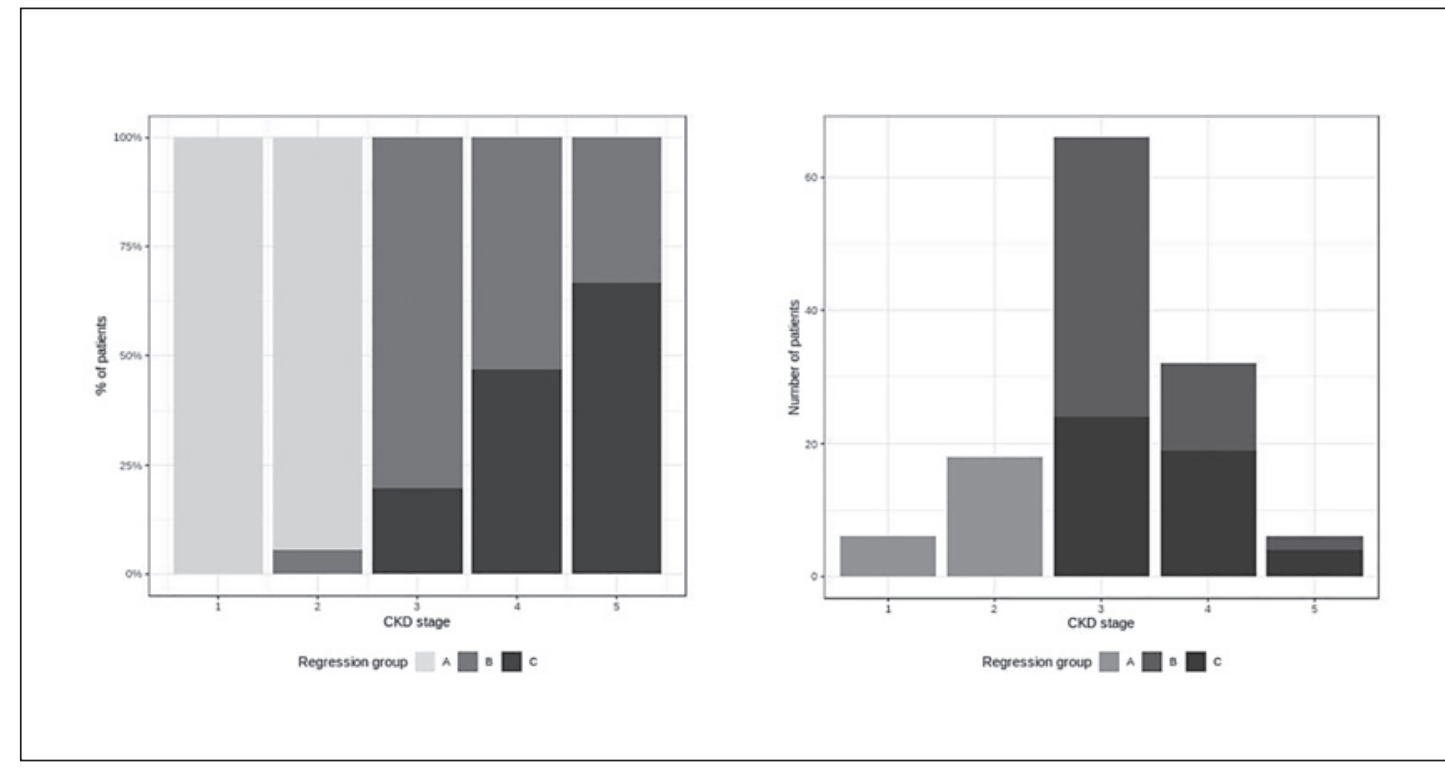

Fig. 1. Correlation of the 5 CKD stages with the 3 regression groups $-\mathrm{A}, \mathrm{B}$, and $\mathrm{C}-$ in percentage (left panel) and in number of patients (right panel) with a test of 3 X 5 contingency table (Pearson's $\chi^{2}$ test, $p<0.0001$ ).

there was a marginal difference in cFGF-23 between them, with lower levels in the group of users $(p=0.045)$.

Classification of Patients according to mGFR Values and Serum Phosphate Levels

Multivariate regression tree analysis using sKlotho as response variable led to the formation of 3 distinct groups $-\mathrm{A}, \mathrm{B}$ and $\mathrm{C}-$ which correlated with CKD stage $(p<0.0001$; Fig. 1), with age (lower in group A as compared to B and C, $p=0.001$ ), and with cFGF-23 and sKlotho ( $p<0.0001$ for both; Fig. 2). A significant difference among the 3 groups was also shown for FEP and for TmP/GFR values ( $p<0.0001$ for both). Higher FEP values characterized regression groups $\mathrm{B}$ and $\mathrm{C}$, compared with group $\mathrm{A}$, while TmP/GFR differed in a biphasic way, with lower values in group B than in groups A and C (Fig. 3). A similar analysis using cFGF-23 as the response variable did not result in similar findings.

\section{Associations of cFGF-23 and sKlotho Plasma Levels with Clinical Outcomes}

Correlation of cFGF-23 and sKlotho with CKD Progression

Univariate linear regression analysis showed that log cFGF-23 and log sKlotho were significantly associated, negatively and positively, with log fGFR, respectively, at the end of the follow-up period ( $p<0.0001$ for both) (Fig. 4). In multiple regression analysis, fGFR was independently associated with mGFR $(p<0.0001)$, sex $(p<0.0001)$, presence of HTN $(p<$ $0.0001)$, iPTH ( $p=0.0003)$, sKlotho $(p=0.002)$, calcium $(p=0.01)$, TmP/GFR $(p=0.01)$, phosphate $(p=0.02)$, high-density lipoprotein $(p=0.03)$, and BMI $(p=0.04)$ (fGFR multiple $R^{2}=$ $0.80, p<0.0001$ ).

Relationship of cFGF-23 and sKlotho with the Primary Endpoint

During the follow-up period (median 36, range 2-60 months), 40 out of the 128 patients (31.2\%) either initiated renal replacement therapy or died (31 and 9, respectively). These 9 deaths were due to cardiovascular causes. Participants with cFGF-23 plasma levels higher than the median value $(218.8 \mathrm{RU} / \mathrm{mL})$ had a shorter event-free survival than those with levels 


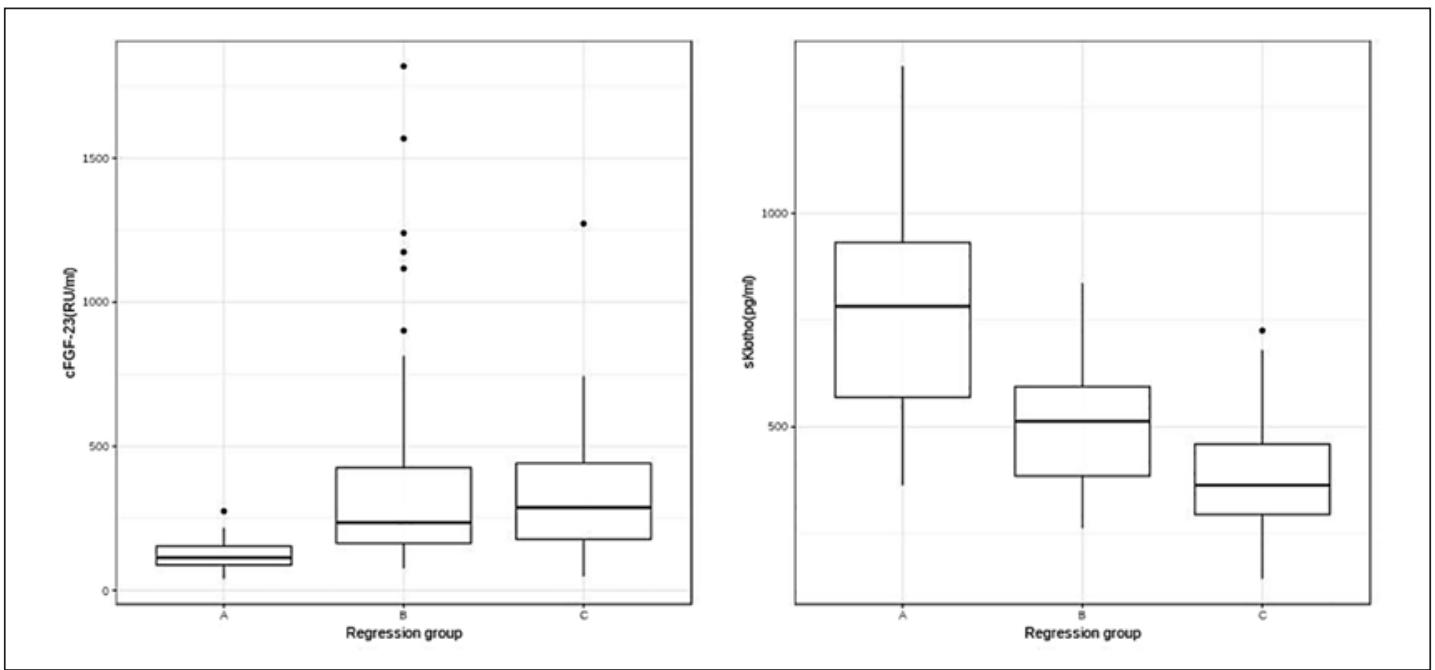

Fig. 2. cFGF-23 (left panel) and sKlotho (right panel) plasma levels differed significantly among the 3 regression groups A, B, and C (Kruskal-Wallis test, $p<0.0001$ for both). cFGF-23, carboxyl terminal fibroblast growth factor 23; sKlotho, soluble Klotho.

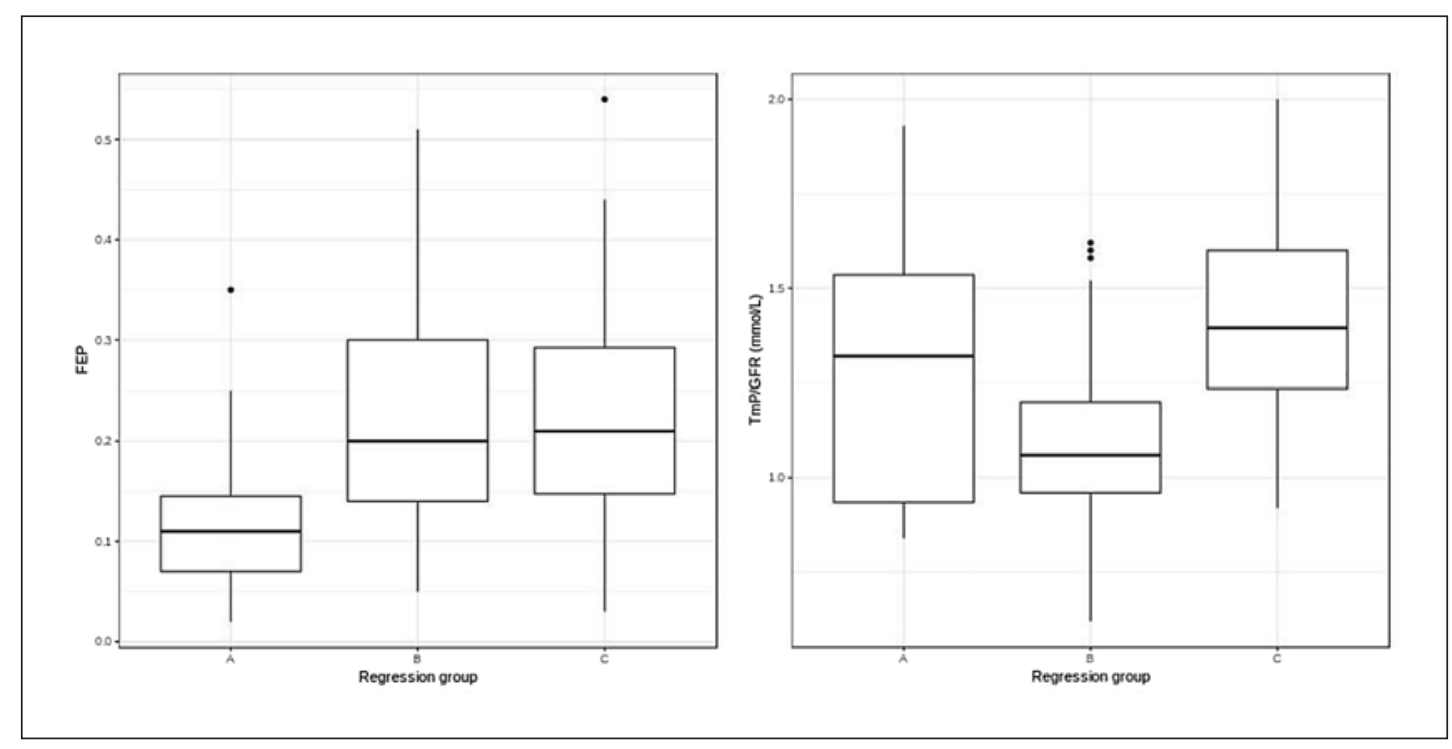

Fig. 3. FEP (left panel) and TmP/GFR (right panel) values differed significantly among the 3 regression groups A, B, and C (Kruskal-Wallis test, $p<0.0001$ for both). FEP, fractional excretion of phosphate; TmP/ GFR, ratio of tubular reabsorption of phosphate to glomerular filtration rate.

below the median ( $p=0.0003)$. Conversely, those with sKlotho plasma levels higher than the median value $(479.8 \mathrm{pg} / \mathrm{mL})$ had a longer event-free survival than those with levels below the median ( $p=0.004$ ) (Fig. 5a). When the cohort was divided by the median value of cFGF-23 and sKlotho plasma levels, the number of composite endpoints was highest in those with high FGF-23/low Klotho $(p<0.0001)$ (Table 2; Fig. 6), with a hazard ratio for the primary outcome of 6.8 (95\% confidence interval [CI] 2.3-19.6; $p=0.0004$ ) compared to those with low FGF-23/ high Klotho. There was no significant event-free survival difference between those with low 


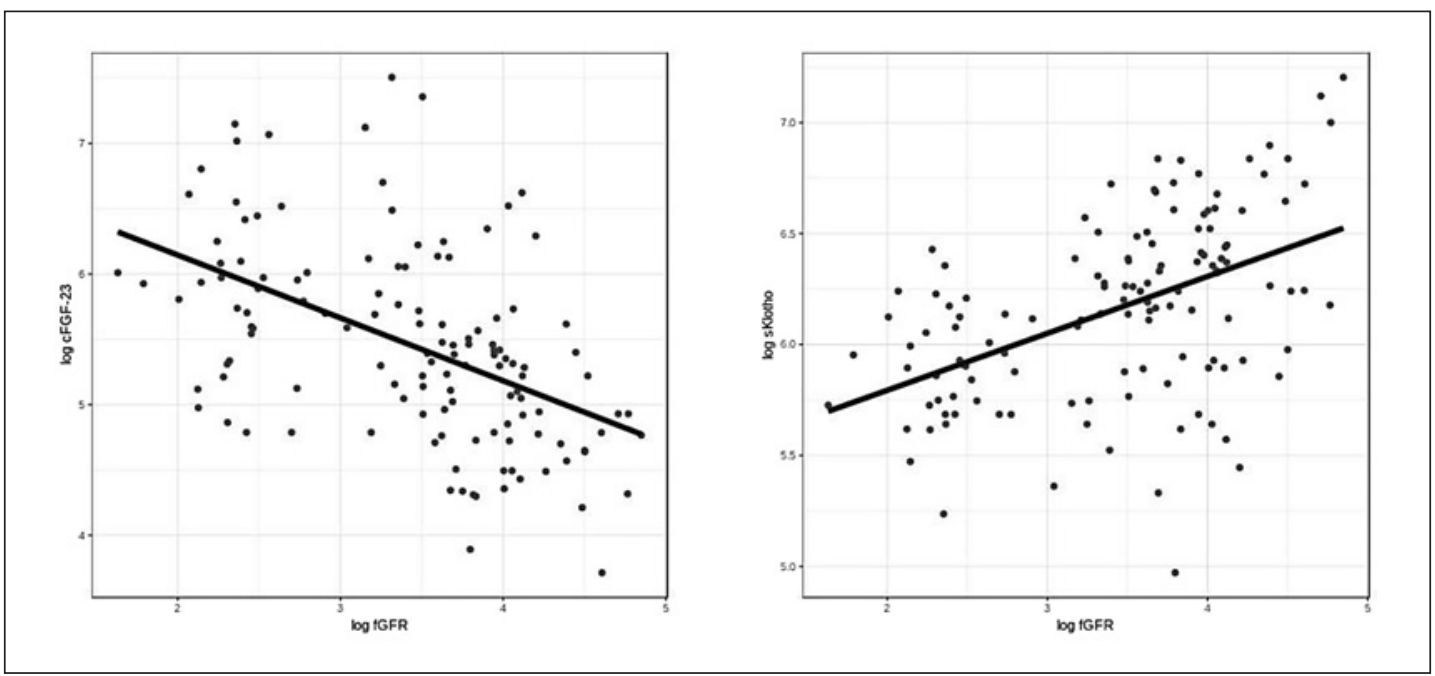

Fig. 4. Univariate linear regression analysis showed that log cFGF-23 (left panel) and log sKlotho (right panel) were significantly correlated with log fGFR value, at the end of follow-up period ( $p<0.0001$ for both). cFGF-23, carboxyl terminal fibroblast growth factor 23; sKlotho, soluble Klotho; fGFR, final glomerular filtration rate.

Table 2. A 2 x 4 contingency table test showing the relationship between combinations of lower or higher than cFGF-23/sKlotho median values with the primary endpoint or event (Pearson's $\chi^{2}$ test, $p<0.0001$ )

\begin{tabular}{lccc}
\hline & Total & No event (0) & Event (1) \\
\hline cFGF-23 lower than median/sKlotho higher than median & 38 & 34 & $4(10.5 \%)$ \\
cFGF-23 lower than median/sKlotho lower than median & 26 & 20 & $6(23 \%)$ \\
cFGF-23 higher than median/sKlotho higher than median & 25 & 18 & $7(28 \%)$ \\
cFGF-23 higher than median/sKlotho lower than median & 39 & 16 & $23(58.9 \%)$ \\
\hline Total & 128 & 88 & $40(31.2 \%)$
\end{tabular}

cFGF-23, carboxyl terminal fibroblast growth factor 23; sKlotho, soluble Klotho.

FGF-23/high Klotho and those with both biomarkers low or both biomarkers high (Table 3 ). Survival curves also differed significantly among the 3 regression groups $\mathrm{A}, \mathrm{B}$, and $\mathrm{C}(p=$ 0.006) (Fig. 5b).

After adjustment for potential confounders (sex, age, BMI, waist-to-hip ratio, presence of DM, history of CVD, hemoglobin, albumin, CRP, ACR, and TmP/GFR), the above model remained significant (Table 4). TmP/GFR, waist-to-hip ratio, and albumin affected significantly the outcome, when they entered the model, but the rest of confounders did not. In particular, patients with higher values of TmP/GFR $(p=0.007)$ showed a more favorable course, while those with higher values of waist-to-hip ratio $(p=0.03)$ showed worse prognosis. Higher values of serum albumin, paradoxically, increased the risk of reaching the primary endpoint $(p=0.03)$.

The slope of the line of GFR versus time decreased in all groups $(p<0.0001)$, regardless of low or high FGF-23 or Klotho combination. However, there was no difference in GFR slope among these 4 groups ( $p=0.656$ ) (Fig. 7). After adjustment for the same potential confounders, a steeper decrease of the slope of the line in group G4 was easily notable for the history of 


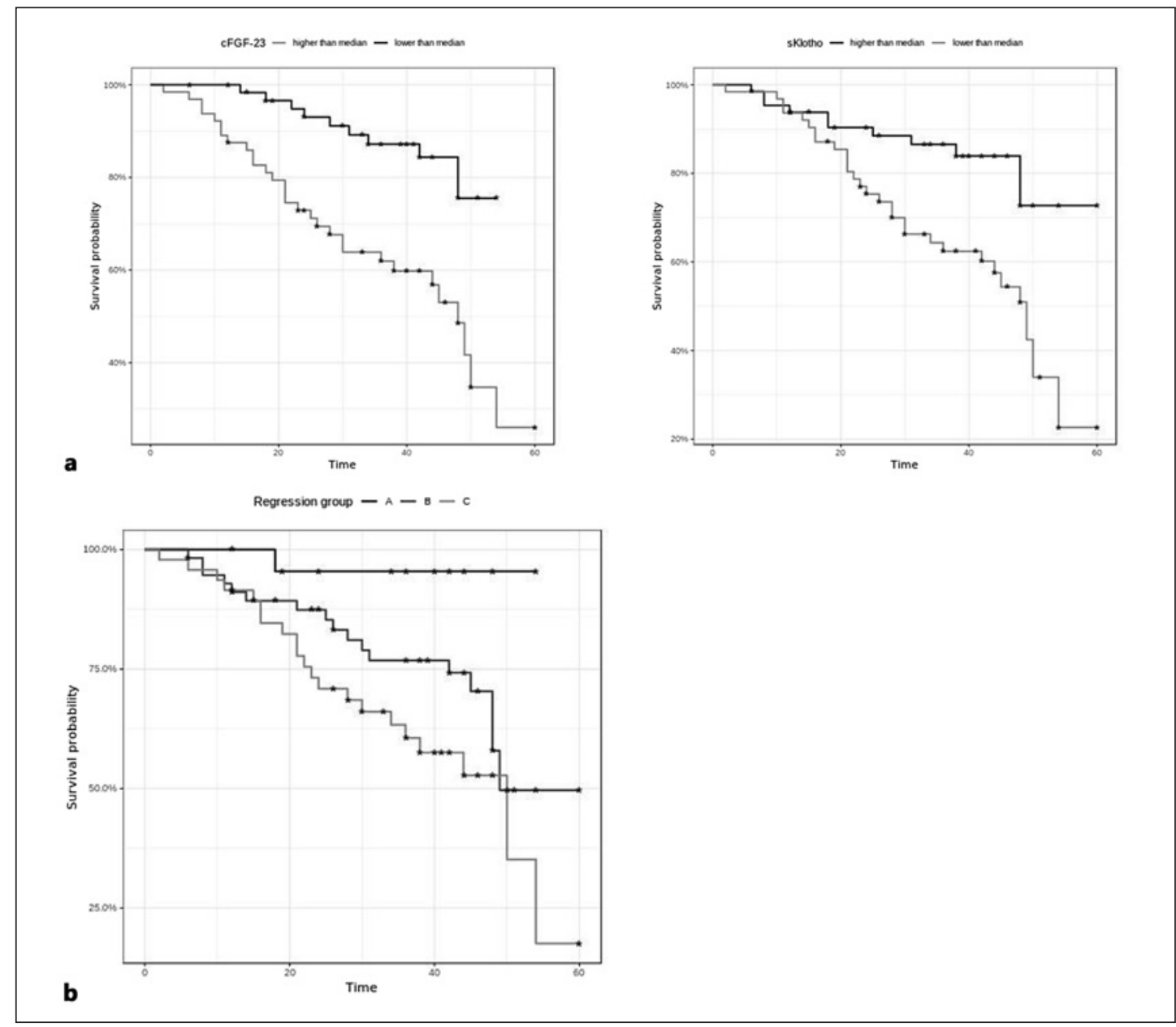

Fig. 5. a The Kaplan-Meier survival analysis showed significant difference, regarding the primary endpoint or event between values lower and higher than median of cFGF-23 (left panel) and sKlotho (right panel) plasma levels ( $p=0.0003$ and $p=0.004$, respectively). Patients with cFGF-23 plasma levels lower than median value (black line) showed better outcome compared with those with higher than median (gray line). On the contrary, patients with sKlotho plasma levels higher than median value (black line) showed better outcome compared with those with lower than median (gray line). b The Kaplan-Meier survival analysis showed significant difference, regarding the primary endpoint or event among the 3 regression groups $\mathrm{A}, \mathrm{B}$, and $\mathrm{C}$ $(p=0.006)$, with the transition from regression group A to groups B and C to be associated with deterioration of outcome. cFGF-23, carboxyl terminal fibroblast growth factor 23; sKlotho, soluble Klotho.

CVD ( $p=0.03)$, TmP/GFR $(p<0.0001)$, waist-to-hip ratio ( $p=0.001)$, age $(p<0.0001)$, and hemoglobin $(p=0.01)$ and marginally for the presence of DM $(p=0.07)$. The rest of confounders, which entered the model, did not give significant results (data are not shown).

\section{Discussion}

It is well known that FGF-23 and $\alpha$-Klotho protein have a major role in regulation of phosphate homeostasis [28] and alterations of their concentrations are the earliest observed abnormalities in CKD-MBD $[3,5]$. In accordance with the above, the present study demon- 
Kidney and

Blood Pressure

Research

Table 3. Cox proportional hazard model for the probability of survival between groups G1-G2, G1-G3, and G1-G4 in a non-adjusted model $(p=0.0002)$

\begin{tabular}{l|l}
\hline Kidney Blood Press Res 2020;45:900-915 \\
\hline DOI: 10.1159/000510351 & $\begin{array}{l}\text { @ 2020 The Author(s). Published by S. Karger AG, Basel } \\
\text { www.karger.com/kbr }\end{array}$ \\
\hline
\end{tabular}

Manou et al.: FGF-23, Klotho, and Adverse Events in Non-Dialysis CKD

\begin{tabular}{lll}
\hline Variable & HR $(95 \% \mathrm{CI})$ & $p$ value \\
\hline G1-G2 & $2.774(0.810-9.491)$ & 0.104 \\
G1-G3 & $2.153(0.607-7.638)$ & 0.235 \\
G1-G4 & $6.775(2.339-19.622)$ & 0.0004 \\
\hline
\end{tabular}

HR $(95 \% \mathrm{CI})$ for primary endpoint related to the combination of cFGF-23/sKlotho in a non-adjusted model Data presented are HR (95\% CI) for primary endpoint related to the combination of cFGF-23/ sKlotho in a non-adjusted model. Group G1, low FGF-23/high Klotho; group G2, low FGF-23/low Klotho; group G3, high FGF-23/high Klotho; group G4, high FGF-23/low Klotho; HR, hazard ratio; CI, confidence interval; FGF-23, fibroblast growth factor 23.
Table 4. Cox proportional hazard model for the probability of survival after adjustment for potential confounders. The primary model was adjusted for sex, age, BMI, waist-to-hip ratio, presence of DM, history of CVD, hemoglobin, albumin, CRP, ACR, and TmP/GFR

\begin{tabular}{lcc}
\hline Variable & HR $(95 \%$ CI $)$ & $p$ value \\
\hline G1-G2 & $3.723(0.965-14.362)$ & 0.056 \\
G1-G3 & $3.391(0.851-13.502)$ & 0.083 \\
G1-G4 & $5.663(1.726-18.581)$ & 0.004 \\
Sex & $0.833(0.312-2.223)$ & 0.716 \\
Age & $0.978(0.943-1.014)$ & 0.237 \\
BMI & $1.005(0.939-1.076)$ & 0.873 \\
Waist-to-hip ratio & $94.527(1.262-7,077.271)$ & 0.038 \\
Presence of DM & $1.048(0.467-2.354)$ & 0.907 \\
History of CVD & $1.567(0.671-3.657)$ & 0.298 \\
Hemoglobin & $0.737(0.529-1.027)$ & 0.072 \\
Albumin & $3.372(1.062-10.702)$ & 0.039 \\
CRP & $1.172(0.963-1.425)$ & 0.112 \\
ACR & $1.000(0.999-1.000)$ & 0.115 \\
TmP/GFR & $0.104(0.020-0.542)$ & 0.007 \\
\hline
\end{tabular}

HR $(95 \% \mathrm{CI})$ for primary endpoint related to the combination of cFGF-23/sKlotho in a non-adjusted model. Group G1, low FGF-23/high Klotho; group G2, low FGF-23/low Klotho; group G3, high FGF-23/high Klotho; group G4, high FGF-23/low Klotho; HR, hazard ratio; CI, confidence interval; BMI, body mass index; DM, diabetes mellitus; CVD, cardiovascular disease; CRP, C-reactive protein; ACR, albumin-to- $\mathrm{Cr}$ ratio; TmP/GFR, ratio of tubular maximum reabsorption of phosphate to glomerular filtration rate; FGF-23, fibroblast growth factor 23.

strated that cFGF-23 plasma levels increased, while those of sKlotho fell significantly from CKD stage 3 and thereafter.

Multivariate regression tree analysis using sKlotho as response variable revealed the formation of 3 distinct groups - regression groups $\mathrm{A}, \mathrm{B}$, and $\mathrm{C}$ - according to mGFR and serum phosphate (cutoff values: $60.85 \mathrm{~mL} / \mathrm{min} / 1.73 \mathrm{~m}^{2}$ and $3.7 \mathrm{mg} / \mathrm{dL}$, respectively). A significant difference of cFGF-23 and sKlotho was observed among the 3 regression groups, although the difference for sKlotho was much more evident than that for cFGF-23. The above relationship among mGFR, phosphate, and sKlotho suggests that sKlotho and phosphate collaborate tightly in CKD progression. Transition from regression group A to groups $\mathrm{B}$ and $\mathrm{C}$ was based only on the level of mGFR, while the difference between group B and group C was further based on serum phosphate level. This observation may demonstrate that even "normal" serum phos- 
Kidney and

Blood Pressure

Research

Fig. 6. The Kaplan-Meier survival analysis revealed a significant difference, regarding the primary endpoint or event, among the 4 groups G1, G2, G3, and G4 ( $p<$ $0.0001)$. The median survival time (50\%) for group G4 appeared to be 44 months, while in the same time, about 94,71 , and $73 \%$ of patients in groups G1, G2, and G3, respectively, were free of event. Group G1, low FGF-23/ high Klotho; group G2, low FGF23/low Klotho; group G3, high FGF-23/high Klotho; group G4, high FGF-23/low Klotho; FGF-23, fibroblast growth factor 23.

Fig. 7. Mixed linear model with repeated measures showed that the height of lines of GFR versus time decreased gradually in the 4 groups $(p<0.0001)$ but without significant difference in the slope among them $(p=0.656)$. Group G1, low FGF-23/high Klotho; group G2, low FGF-23/low Klotho; group G3, high FGF-23/high Klotho; group G4, high FGF-23/ low Klotho; FGF-23, fibroblast growth factor 23 .

\begin{tabular}{l|l}
\hline Kidney Blood Press Res 2020;45:900-915 \\
\hline DOI: 10.1159/000510351 & $\begin{array}{l}\text { ○ 2020 The Author(s). Published by S. Karger AG, Basel } \\
\text { www.karger.com/kbr }\end{array}$ \\
\hline
\end{tabular}

Manou et al.: FGF-23, Klotho, and Adverse Events in Non-Dialysis CKD
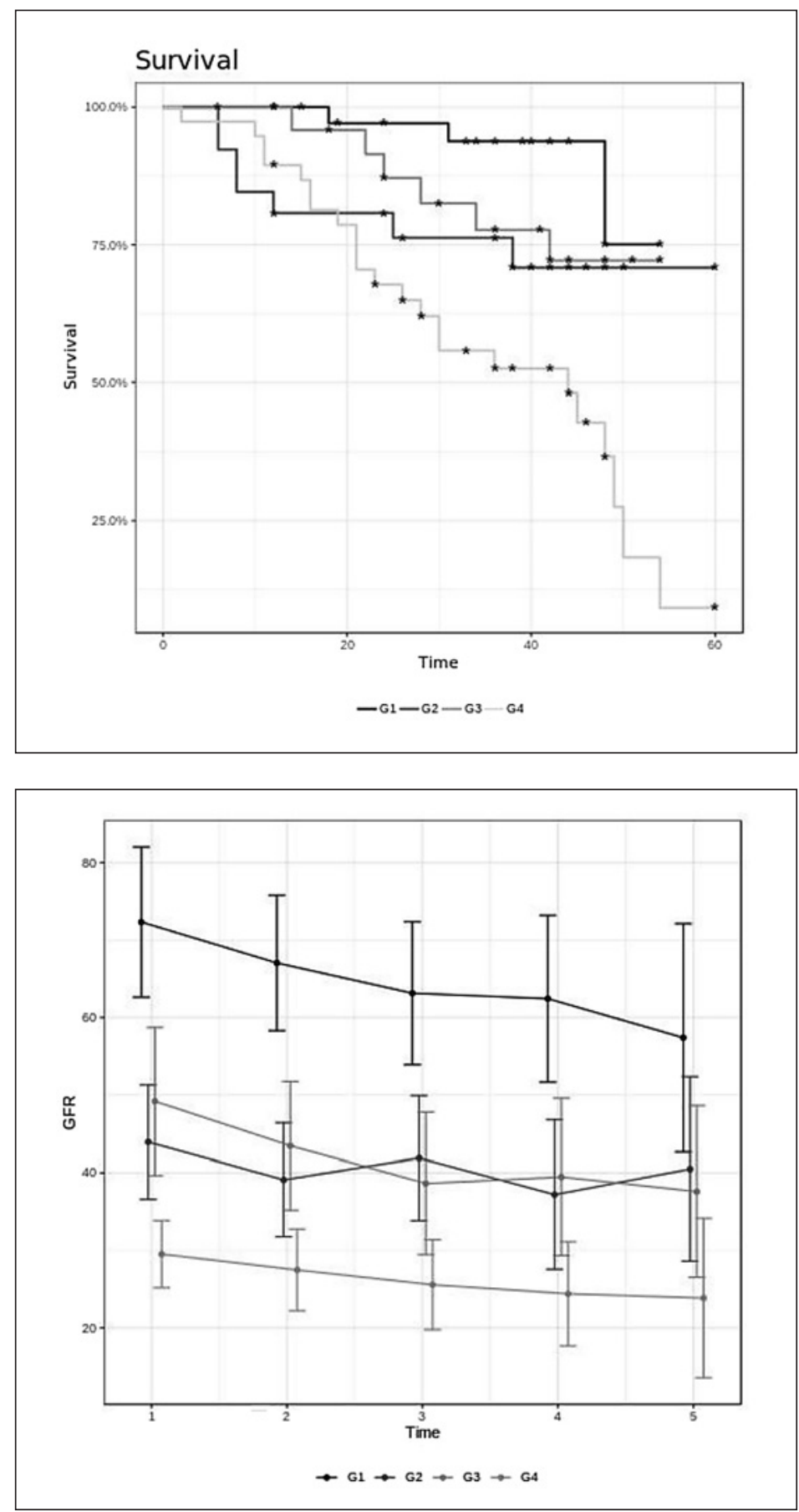

phate, such as the value of $3.7 \mathrm{mg} / \mathrm{dL}$, could be combined on the one hand with more advanced CKD stages and on the other hand with lower sKlotho and higher cFGF-23 levels. This interplay between sKlotho and phosphate in CKD has gained interest in the field of CKD-MBD and may be indicative of the need for early intervention in the regulation of serum phosphate levels, even when these are within normal limits [29]. Furthermore, we observed that when serum phosphate was equal or $>3.7 \mathrm{mg} / \mathrm{dL}$ in conjunction with a reduced mGFR of $<60.85 \mathrm{~mL} /$ $\mathrm{min} / 1.73 \mathrm{~m}^{2}$, as in the case of regression group C, the value of sKlotho was in its lowest level. On the contrary, cFGF-23 plasma levels in regression group C did not show a "clear" rise 
compared with those in the previous regression group $B$, but only with those in the regression group A, which included patients with preserved mGFR. The above sorting of patients, according to mGFR and serum phosphate, which highly correlates with the classic CKD staging, and its close association mainly with sKlotho and secondarily with cFGF-23, may denote that the role of Klotho protein, compared with that of FGF-23, may be more decisive to renal phosphate handling in CKD patients. Moreover, this role is not clear enough, as experimental studies have shown Klotho's phosphaturic action, while other studies in CKD patients have argued for a positive role of sKlotho in phosphate reabsorption $[18,30]$.

Log sKlotho correlation with log fGFR remained significant in multiple regression analysis after adjustment for several confounders, known to be associated with CKD progression. The association of FGF-23 with CKD progression has been previously reported; however, the number of studies investigating the prognostic value of sKlotho is very limited and their results are contradictory $[19,20]$. In a recent meta-analysis of 8 cohort studies, the relationship between sKlotho and risk of adverse renal outcomes in CKD patients was examined, finding out that low sKlotho is significantly associated with an increased risk of poor renal outcomes [31]. However, there are many conflicting results about Klotho that require further studies.

cFGF-23 and sKlotho together affected patients' outcome, as this was shown by the different percentage of patients reaching the primary endpoint in the 4 groups, based on division of the cohort by median values of both biomarkers. The median event-free survival time was significantly shorter in the group G4 than in the rest, with patients in this group having the highest likelihood of presenting with an event. After adjustment for potential confounders, the Cox regression model remained significant. The paradox of the higher risk of an event when serum albumin was elevated may be due to the majority of albumin levels in patients' serum within the normal range. In addition, waist-to-hip ratio, as well as phosphaturia, also increased the risk. The Kaplan-Meier survival analysis evaluating the 2 biomarkers separately showed that patients with cFGF-23 levels more than median and those with sKlotho levels less than median value showed a worse outcome in comparison with the opposite ones. The same was observed among the 3 regression groups $\mathrm{A}, \mathrm{B}$, and $\mathrm{C}$, with a deterioration of survival from $\mathrm{A}$ to $\mathrm{C}$ group. This may denote that the tree analysis classification of our patients is an interesting finding, since not only mGFR, serum phosphate, and sKlotho plasma levels are related to this classification, but also the outcome of the patients.

The height of lines of GFR versus time for the groups G1, G2, G3, and G4 showed a relative gradual reduction. However, no difference was observed among the 4 groups in how quickly the values of GFR declined over time. The unadjusted mixed linear model failed to confirm the results of the survival analyses. In other words, although the likelihood of presenting with an event was more prominent in the group G4, the above model did not reveal that GFR loss was faster in this group than in the others. We argue that these somewhat unclear and contradictory data do not reduce the magnitude of both FGF-23 and sKlotho's contribution to the outcome, but are rather indicative of a multifactorial nature of this process. It is well known that many other traditional and CKD-related factors may play a role affecting patients' outcome. In addition, the adjusted analyses of mixed linear model showed that the presence of some variables aggravated GFR loss in group G4, supporting this multifactorial nature.

All the above results underline the important role of FGF-23/sKlotho not only in CKD progression but in cardiovascular mortality too. Of note, several studies have shown an independent correlation of FGF-23 with all-cause mortality, but those assessing cardiovascular mortality are limited $[7,9,12,13]$. Additionally, there are only 2 previous studies in hemodialysis patients, which showed a correlation of sKlotho levels with all-cause and cardiovascular mortality $[32,33]$ and only one in non-dialysis patients with CKD stages 2-4, in whom no association between sKlotho and cardiovascular outcomes was found [34]. 
In our study, we examined the role of cFGF-23 and sKlotho in risk stratification in CKD. The evaluation of contribution of these 2 biomarkers simultaneously in renal clinical outcomes has not been extensively reviewed in previous studies. The main limitation of our study is the relatively small patient number and the small number of cardiovascular deaths, during the follow-up period. In addition, cFGF-23 and sKlotho were measured only at baseline, with no further assessment. However, our results are strengthened by the large number of measured clinical and laboratory parameters, known to affect outcome in CKD patients, the extensive statistical adjustment, and the adequate follow-up period.

\section{Conclusions}

In patients with CKD stages 1-5, cFGF-23 and sKlotho levels are independently associated with progression to end-stage kidney disease and cardiovascular mortality. In addition, mGFR, serum phosphate, and sKlotho plasma levels seem to have a synergistic and a prognostic role in the outcome of these patients. Studies are required to determine whether modification of their levels toward "normal," through different therapeutic approaches, would improve clinical outcomes in this high-risk patient population.

\section{Acknowledgement}

The authors wish to thank Dr. Stavros Panidis, for his contribution to the statistical processing of the study and the analysis of the results.

\section{Statement of Ethics}

The study protocol was approved by the Institutional Ethics Committee of the Papageorgiou General Hospital of Thessaloniki, and all participants gave a written informed consent. All procedures were conducted in accordance with the Declaration of Helsinki (2008 Amendment).

\section{Conflict of Interest Statement}

All the authors declared no conflicts of interest.

\section{Funding Sources}

All the authors declared no funding sources.

\section{Author Contributions}

E.M. was responsible for the execution of the study protocol, the gathering and statistical evaluation of the results, and the writing the manuscript. She is the corresponding author. E.T. was involved in conceiving the main study idea and contributed to the evaluation of the results and the revision of the manuscript. G.A. performed main study and contributed to the 
evaluation of the results and the revision of the manuscript. P.P. contributed to the evaluation of the results and the revision of the manuscript. S.P. contributed to the evaluation of the results and the revision of the manuscript. D.P. contributed to the evaluation of the results and the revision of the manuscript. A.P. was involved in conceiving the main study idea and contributed to the evaluation of the results and the revision of the manuscript. All authors discussed the results and contributed to the final manuscript.

\section{References}

1 KDIGO Clinical practice guideline for the diagnosis, evaluation, prevention, and treatment of Chronic Kidney Disease-Mineral and Bone Disorder (CKD-MBD). Kidney Int Suppl. 2009;76(113):S1-130.

2 Isakova T, Wahl P, Vargas G, Gutiérrez OM, Scialla J, Xie H, et al. FGF23, PTH and phosphorus metabolism in the chronic renal insufficiency cohort. Kidney Int. 2011;79(12):1370-8.

3 Pavik I, Jaeger P, Ebner L, Wagner CA, Petzold K, Spichtig D, et al. Secreted Klotho and FGF23 in chronic kidney disease stage 1 to 5: a sequence suggested from a cross-sectional study. Nephrol Dial Transplant. 2013;28(2): 352-9.

4 Kovesdy CP, Quarles LD. Fibroblast growth factor-23: what we know, what we don't know, and what we need to know. Nephrol Dial Transplant. 2013;28(9):2228-36.

5 Wolf M. Forging forward with 10 burning questions on FGF23 in kidney disease. J Am Soc Nephrol. 2010; 21(9):1427-35.

6 Wesseling-Perry K, Jüppner H. The osteocyte in CKD: new concepts regarding the role of FGF23 in mineral metabolism and systemic complications. Bone. 2013;54(2):222-9.

7 Kendrick J, Cheung AK, Kaufman JS, Greene T, Roberts WL, Smits G, et al. FGF-23 associates with death, cardiovascular events, and initiation of chronic dialysis. J Am Soc Nephrol. 2011;22(10):1913-22.

8 Wolf M, Molnar MZ, Amaral AP, Czira ME, Rudas A, Ujszaszi A, et al. Elevated fibroblast growth factor 23 is a risk factor for kidney transplant loss and mortality. J Am Soc Nephrol. 2011;22(5):956-66.

9 Gutiérrez OM, Januzzi JL, Isakova T, Laliberte K, Smith K, Collerone G, et al. Fibroblast growth factor 23 and left ventricular hypertrophy in chronic kidney disease. Circulation. 2009;119(19):2545-52.

10 Jovanovich A, Ix JH, Gottdiener J, McFann K, Katz R, Kestenbaum B, et al. Fibroblast growth factor 23, left ventricular mass, and left ventricular hypertrophy in community-dwelling older adults. Atherosclerosis. 2013;231(1):114-9.

11 Gutiérrez OM, Mannstadt M, Isakova T, Rauh-Hain JA, Tamez H, Shah A, et al. Fibroblast growth factor 23 and mortality among patients undergoing hemodialysis. N Engl J Med. 2008;359(6):584-92.

12 Jean G, Terrat JC, Vanel T, Hurot JM, Lorriaux C, Mayor B, et al. High levels of serum fibroblast growth factor (FGF)-23 are associated with increased mortality in long haemodialysis patients. Nephrol Dial Transplant. 2009;24(9):2792-6.

13 Souma N, Isakova T, Lipiszko D, Lipiszko D, Sacco RL, Elkind MS, et al. Fibroblast growth factor 23 and cause specific mortality in the general population: the Northern Manhattan Study. J Clin Endocrinol Metab. 2016; 101(10):3779-86.

14 Haring R, Enserro D, Xanthakis V, Mitchell GF, MD, Hamburg NM, et al. Plasma fibroblast growth factor 23: clinical correlates and association with cardiovascular disease and mortality in the Framingham Heart Study. J Am Heart Assoc. 2016;5(7):e003486.

15 Kuro-o M. Klotho and aging. Biochim Biophys Acta. 2009;1790(10):1049-58.

16 Hu MC, Kuro-o M, Moe OW. Secreted Klotho and chronic kidney disease. Adv Exp Med Biol. 2012;728:126-57.

17 Hu MC, Kuro-o M, Moe OW. The emerging role of Klotho in clinical nephrology. Nephrol Dial Transplant. 2012; 27(7):2650-7.

18 Hu MC, Shi M, Zhang J, Pastor J, Nakatani T, Lanske B, et al. Klotho: a novel phosphaturic substance acting as an autocrine enzyme in the renal proximal tubule. FASEB J. 2010;24(9):3438-50.

19 Kim HR, Nam BY, Kim DW, Kang MW, Han JH, Lee MJ, et al. Circulating $\alpha$-klotho levels in CKD and relationship to progression. Am J Kidney Dis. 2013;61(6):899-909.

20 Seiler S, Wen M, Roth HJ, Fehrenz M, Flügge F, Herath E, et al. Plasma Klotho is not related to kidney function and does not predict adverse outcome in patients with chronic kidney disease. Kidney Int. 2012;83(1):121-8.

21 K/DOQI clinical practice guidelines for chronic kidney disease: evaluation, classification and stratification. Kidney Disease Quality Initiative. Am J Kidney Dis. 2002;39(2):S1-266.

22 Haycock GB, Schwartz GJ, Wisotsky DH. Geometric method for measuring body surface area: a height-weight formula validated in infants, children, and adults. J Pediatr. 1978;93(1):62-6.

23 Walton RJ, Bijvoet OL. Nomogram for derivation of renal threshold phosphate concentration. Lancet. 1975; 2(7929):309-10.

24 Fleming JS, Zivanovic MA, Blake GM, Burniston M, Cosgriff PS. Guidelines for the measurement of glomerular filtration rate using plasma sampling. Nucl Med Commun. 2004;25(8):759-69. 
25 Mortensen JB, Rödbro P. Comparison between total and renal plasma clearance of [51Cr] EDTA. Scand J Clin Lab Invest. 1976;36(3):247-9.

26 Levey AS, Stevens LA, Schmid CH, Zhang YL, Castro AF, Feldman HI, et al. A new equation to estimate glomerular filtration rate. Ann Intern Med. 2009;15(9):604-12.

27 Morgan J. Classification and regression tree analysis. Technical report no. 1. Boston University School of Public Health; 2014.

28 Razzaque MS. The FGF23-Klotho axis: endocrine regulation of phosphate homeostasis. Nat Rev Endocrinol. 2009;5(11):611-9.

29 Cannata-Andía JB, Martin KJ. The challenge of controlling phosphorus in chronic kidney disease. Nephrol Dial Transplant. 2016;31(4):541-7.

30 Tan SJ, Smith ER, Holt SG, Hewitson TD, Toussaint ND. Soluble klotho may be a marker of phosphate reabsorption. Clin Kidney J. 2017;10(3):397-404.

31 Liu QF, Yu LX, Feng JH, Sun Q, Li SS, Ye JM. The Prognostic role of Klotho in patients with chronic kidney disease: a systematic review and meta-analysis. Dis Markers. 2019;2019:6468729.

32 Marçais C, Maucort-Boulch D, Drai J, Dantony E, Carlier MC, Blond E, et al. Circulating Klotho associates with cardiovascular morbidity and mortality during hemodialysis. J Clin Endocrinol Metab. 2017;102(9):3154-61.

33 Otani-Takei N, Masuda T, Akimoto T, Honma S, Watanabe Y, Shiizaki K, et al. Association between serum soluble Klotho levels and mortality in chronic hemodialysis patients. Int J Endocrinol. 2015;2015:406269.

34 Seiler S, Rogacev KS, Roth HJ, Shafein P, Emrich I, Neuhaus S, et al. Associations of FGF-23 and sKlotho with cardiovascular outcomes among patients with CKD stages 2-4. Clin J Am Soc Nephrol. 2014;9(6):1049-58. 